\title{
Artificial Bee Colony for Quadratic Assignment Problem: A Hospital Case Study
}

\author{
Jalal A. Sultan \\ Department of Mathematics, \\ College of Education for Pure Sciences, \\ University of Mosul, Iraq \\ Jalalstat2011@gmail.com
}

\author{
Daham A. Matrood \\ College of Mathematics and \\ Computer Sciences, \\ University of Mosul, Iraq \\ dham.auid@gmail.com
}

\author{
Zaidoun M. Khaleel \\ College of Mathematics and \\ Computer Sciences, \\ University of Mosul, Iraq \\ zekh97@gmail.com
}

\begin{abstract}
The problem of locating hospital departments so as to minimize the total distance travelled by patients can be formulated as a Quadratic Assignment Problem (QAP).In general, (QAP) is one of the Combinatorial Optimization Problems and always high dimensional. Therefore, the use of meta-heuristics that generates good solutions in reasonable computer time becomes an attractive alternative. In this paper, a proposed artificial bee colony (ABC) algorithm is used to optimize QAP. The main idea is to use different crossover techniques for employee and onlooker bee stages and use exchange position operator for scout bee stage. The results of $\mathrm{ABC}$ algorithm show the efficiency and capabilities of proposed algorithm in finding the optimum solutions, compared with results of GA and SA in all test problems. The purpose of this paper is to apply the QAP in Azadi hospital in Kirkuk city to minimize the total distance travelled by patients. The application involves determine the flow matrix and the distance matrix to solve the problem. The results related that QAP model was presented suitable framework for clinics allocation and optimum use.
\end{abstract}

Keywords- Quadratic Assignment Problem; Artificial Bee Colony; Hospital Layout; Healthcare Management.

\section{INTRODUCTION}

There are many scientific questions raised "What is the optimal way to wire a computer backboard?", "How are the locations of clinics within a hospital decided?", " How is deciding on the sites of colleges and departments on campus? ","How was the access to the current arrangement of the keyboard?". What possible linkages could there be between these problems? Most would agree that at first glance, they are seemingly unrelated beyond the fact that both are decision problems. One might even propose that such decisions are made arbitrarily. However, it is the solution to these and countless other problems that contains the key to their correlation. They are all modeled by one of the most challenging problems in combinatorial optimization $[23,25]$. This problem has been a focus of researchers for over five decades; it is known as the Quadratic Assignment Problem [6].

The Quadratic Assignment Problem (QAP) was originally introduced in 1957 by Koopmans and Beckman who were trying to model a facilities location problem. Since then, it has been among the most studied problems in all of combinatorial optimization. Many scientists including mathematicians, computer scientists, operations research analysts, and economists have used the QAP to model a variety of optimization problems [18].

In general, Quadratic Assignment problems are NP-hard problems, that is, unlikely there is no an algorithm that can find an optimal QAP solution in polynomial time. In practical, this means that the processing time required to solve such problem will quickly and enormously grow as the problem size grows. For this reason, truly optimal solution is quite difficult to be found. Therefore, meta-heuristic techniques such as genetic algorithm, simulated annealing, tabu search, artificial bee colony etc. are also employed to obtain optimum solution [4,21].

In general, the main advantages of meta-heuristic are: they do not require the objective function to be differentiable or continuous, they do not require the evaluation of gradients, and they can escape from local minima [2].

In this paper, a proposed artificial bee colony proposed to solve quadratic assignment problems. The purpose of this paper is to apply QAP in a public hospital in Iraq. The application involves determine the flow matrix and the distance matrix to solve the problem.

The paper is organized as follows: In section 2 the main idea for QAP is presented as well as the mathematical formulation. The ABC algorithm to solve QAP problems has been proffered in Section 3. In section 4 numerical experiments and the Apply of QAP for Azadi hospital is presented in section 5. The last section concludes the paper.

\section{A BIT FROM QAP AND ABC}

\section{A. Quadratic Assignment Problem}

Quadratic Assignment Problem (QAP) is one of the challenging classical combinatorial optimization problems. QAP was presented by Koopman and Beckman in 1957 [18]. It is a model for many practical problems like backboard wiring, campus and hospital layout, and scheduling [6, 9, 23, 25].

To have an instance of QAP, the full list of distances among available locations $\left(d_{r s}\right)$ and material flow among facilities $\left(F_{i j}\right)$ and should 
be visible. There are $\mathrm{N}$ facilities and each of them can be interchanged with each other. On the other hand, there are $\mathrm{N}$ locations each of them can be provided for only one facility. Hence, the QAP can be modeled as follows [20]:

There are a set of $\mathrm{N}$ facilities and a set of $\mathrm{N}$ locations. For each pair of locations, a distance $\left(d_{i j}\right)$ is specified and for each pair of facilities a flow $\left(F_{i j}\right)$ is specified. The problem is to assign all facilities to different locations with the goal of minimizing the sum of the distances multiplied by the corresponding flows. Formally, let $d_{i j}$ and $F_{i j}$ be two N*N matrices and let $P_{n}$ be the set of permutation of $\{1,2, \ldots, N\}$.

Then, the QAP can be defined as follows:

$$
\min z=\sum_{i=1}^{n} \sum_{j=1}^{n} f_{i j} d_{\varphi(i) \varphi(j)}
$$

Where $\varphi$ is a vector of permutations possible $(1,2, \ldots, n)$, and that $\varphi(i)$ refers to the site which will be allocated to facilitate $i$, and in the end is the best solution of the problem, Each individual product $f_{i j} d_{\varphi(i) \varphi(j)}$ is the transportation cost caused by assigning facility i to location $\varphi$ (i) and facility j to location $\varphi$ (j).

Figure 1 and Figure 2 shows a map that 6 different facilities located on 6 different locations.

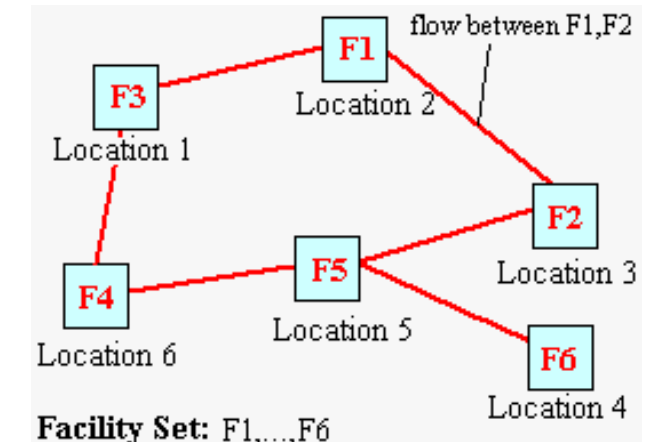

Figure 1: Assigning 6 Facilities to 6 Locations

Here, is a permutation that gives formal definition of Figure 1.

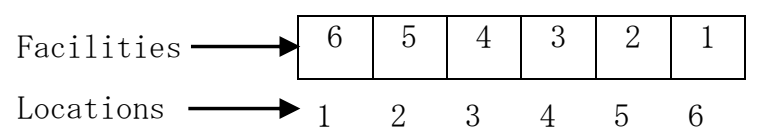

Figure 2: Permutation for Figure 1
According to the allocation in Figure 2, facility 5 has been assigned to location 4, facility 6 to location 1 and so on. The fitness function (f) of this example can be calculated as follows:

$$
Z=\sum_{i=1}^{6} \sum_{j=1}^{6} f_{i j} d_{\varphi(i) \varphi(j)}
$$

The most important and used methods to achieve optimality for QAP are the Branch and bound and cutting plane as they are most efficient techniques. The Branch and bound procedures was firstly introduced by Gilmore [11] in 1962 who solved a QAP of size $8=\mathrm{n}$. Traditional cutting plane algorithms for QAP first used by Kaufman and Broekx in 1978 [3].

Because of obvious difficulties experienced in the development of exact solution procedures, a wide variety of heuristic approaches has been developed for QAP. Heuristic algorithms do not give a guarantee of optimality for the best solution obtained. In this context, we consider heuristic techniques as a procedure dedicated to the search of good quality solutions. These approaches can be classified into the following categories: Construction methods [1] , simulated annealing[20], Genetic algorithms[28], Greedy randomized adaptive search procedures [19], and Ant colonies [27] and Artificial bee colony [4].

Over the years, the QAP has drawn the researcher's attention worldwide and extensive research has been done. From the theoretical point of view, it is because of the high computational complexity: QAP is NP-hard, and even finding an approximate solution is a hard problem [7]. Moreover, many well-known classical combinatorial optimization problems such as the traveling salesman problem, the graph-partitioning problem, the maximum clique problem can be reformulated as special cases of the QAP. From the practical point of view, it is because of the diversified applications of the QAP. The QAP has been applied in many fields such as backboard wiring [18], typewriter keyboards and control panels design [23], scheduling [5], and many others.

\section{B. Artificial Bee Colony}

By simulating the forging behavior of bee colonies, artificial bee colony (ABC) algorithm, which is a swarm intelligence-based optimization algorithm, was proposed by Karaboga in (2005) for numerical function optimization [15]. The main steps of ABC algorithm can be described as follows.

\section{Initialization}

Repeat

Employed bee stage: Place the employed bees on the food sources in the memory.

Onlooker bee stage: Place the onlooker bees on the food sources in the memory. 
Scout bee stage: Send the scout bees to the search area for discovering new food sources.

\section{Until (conditions are satisfied)}

In $\mathrm{ABC}$ algorithm, the colony consists of three kinds of bees: employed bees, onlooker bees and scout bees. Half of the colony is employed bees, and the other half is onlooker bees. The employed bees explore the food source and send the information of the food source to the onlooker bees. The onlooker bees choose a food source to exploit based on the information shared by the employed bees. The scout bee, which is one of the employed bees whose food source are abandoned, finds a new food source randomly. The position of a food source is a possible solution to the optimization problem. Denote the food source number as SN, the position of the $i^{\text {th }}$ food source as $\operatorname{xi}(\mathrm{i}=1, \ldots, \mathrm{SN})$, which is a $\mathrm{D}$ dimensional vector $[14,16]$.

In $\mathrm{ABC}$ algorithm, the $\mathrm{i}^{\text {th }}$ fitness value $\mathrm{i}$ fit for $\mathrm{a}$ minimization problem is defined as [13]:

$$
\text { fit }_{\mathrm{i}}=\left\{\begin{array}{c}
1 /\left(1+f_{i}\right) \text { if } f_{i} \geq 0 \\
1+\operatorname{abs}\left(f_{\mathrm{i}}\right) \text { if } f_{i}<0
\end{array}\right.
$$

Where $f_{i}$ is the cost value of the ith solution. The probability that food source being $\mathrm{j}^{\text {th }}$ selected by an onlooker bee is given by:

$$
p_{i}=\frac{f i t_{i}}{\sum_{i=1}^{S N} f i t_{i}}
$$

A candidate solution from the old one can be generated as:

$$
v_{i j}=x_{i j}+\phi_{i j}\left(x_{i j}-x_{k j}\right)
$$

Where $k \in\{1,2, \ldots, S N\}, k \neq i \cdots$ and $j \in\{1,2, \ldots, D\}$ are randomly selected indices, $\phi_{i j} \in[-1,1]$ is a uniformly distributed random number. The candidate solution is compared with the old one, and the better one should be remained [16].

If the abandoned food source is $\mathrm{xi}$, the scout bee exploits a new food source according to:

$$
x_{i j}=x_{\text {min, } \mathrm{j}}+\operatorname{rand}(0,1)\left(x_{\max , \mathrm{j}}-x_{\min , \mathrm{j}}\right)
$$

Where $x_{\max , \mathrm{j}}$ and $x_{\min , \mathrm{j}}$ are the upper and lower bounds of the $\mathrm{j}^{\text {th }}$ dimension of the problem's search space [14].

\section{PROPOSED ABC To SOLVE QAP}

In this section, we describe the $\mathrm{ABC}$ algorithm for solving QAP problems.

Step 1: Initializing parameters of ABC algorithm and set $\mathrm{Nd}=0$;

Step 2:

2.1: Define the matrices of QAP;

2.2: Generate initial population;

2.3: Evaluate the fitness value of the initial population;

Step 3: Decade loop $\mathrm{Nd}=\mathrm{Nd}+1$
Step 4:

4.1: Search the new food source for employed

bee;

4.2: Apply a greedy selection process;

4.3: If solution does not improve trail $l_{i}=$ trail $_{i}$

+1 , otherwise trail $=0$;

4.4 Apply roulette wheel selection to choose a

food source for onlooker bees;

4.5 Search the new food source for onlooker

bees;

4.6: Apply a greedy selection process;

4.7: If solution does not improve trail $_{i}=$ trail $_{i}$ +1 , otherwise $\operatorname{trail}_{i}=0$;

4.8: If $\max \left(\operatorname{trail}_{i}\right.$ ) > limit, replace this food

source with a new food source produced;

Step 5: Terminating Criterion Control; Repeat Steps 3-5 until a terminating criterion is satisfied;

\section{A. Input Information}

For QAP, the software implemented takes into consideration the following matrices:

- $\quad \mathrm{F}=\left(f_{i j}\right)$, where $f_{i j}$ is the flow from facility $\mathrm{i}$ to facility $\mathrm{j}$;

- $\quad \mathrm{D}=\left(\mathrm{d}_{i j}\right)$, where $d_{i j}$ is the distance from location $\mathrm{i}$ to location $\mathrm{j}$;

\section{B. Fitness Function}

Fitness function is a critical factor in the $\mathrm{ABC}$ Algorithm. Every Source of food in the ABC's population has a fitness value, and it moves in solution space with respect to its previous position where it has met the best fitness value. In this paper, the fitness value is the cost function of QAP denoted as eq. (1) that must be minimized through the iteration process.

\section{Generate an Initial Solution}

The encoding strategy for the QAP, which is one of the combinatorial optimization problems, the sequential encoding type is employed here. Then, each food source can be randomly generated an initial solution for the problem which assigns each department at exactly one dimension. Figure 3 presents the encoding of QAP for the 30 departments, which shows the department 14 at the first position, department 5 at the second position, and so on. Thus, the initial solution is done after we deal with the encoding and to generate initial solution.

\begin{tabular}{|l|l|l|l|l|l|l|l|}
\hline 14 & 5 & 28 & 24 & $\ldots$ & 12 & 11 & 23 \\
\hline
\end{tabular}

The initialization phase is continued for FN times. FN is equal to the half of SN (the bees' population). The best solution is taken from this step and GB keeps it. Also the Extent Memory (EA) keeps the half best solutions in this phase.

\section{Search Mechanism}

It is well known that both the exploration and exploitation abilities are necessary for the population based algorithms. How to balance these two abilities to achieve good optimization performance is very important. 
The original version of $\mathrm{ABC}$ operates on real values. But QAP problem is a discrete problem. So we use new methods for the employed bee stage and the onlooker bee stage which are suitable for the problem.

In the employed bee stage, an example of this operator is shown in figure 4. This operator is as below: 1) Choose some cells (about $50 \%$ of cells) randomly in food source array (cells $\{1,6,2,8$ and 7$\}$ )

2) Copy selected cells into new food source array.

3) Scan whole of food source array from first to end. If there is any cell which is not in food source, copy it in to first empty cell (cells $\{5,10,3,4$ and 9$\}$ ).

\begin{tabular}{|l|c|c|c|c|c|c|c|c|c|c|}
\hline Food $_{i}$ & 1 & 5 & 6 & 2 & 9 & 10 & 3 & 8 & 4 & 7 \\
\hline Food $_{k}$ & 2 & 5 & 10 & 7 & 8 & 3 & 1 & 4 & 6 & 9 \\
\hline New Food & 1 & 5 & 6 & 2 & 10 & 3 & 4 & 8 & 9 & 7 \\
\hline
\end{tabular}

Figure 4. An example of employed bee stage operator

The onlooker bee stage represents PMX (Partial Message Crossover). There are two versions of the method, including two point crossover and single point crossover. The PMX process is as follows :

Step 1: Select two parents P1 and P2 randomly from

\begin{tabular}{|c|c|c|c|c|c|c|c|}
\hline $\boldsymbol{i}$ & Problem & $\boldsymbol{N}$ & $\begin{array}{c}\text { Optim } \\
\boldsymbol{a l}\end{array}$ & $\boldsymbol{i}$ & $\begin{array}{c}\text { Proble } \\
\boldsymbol{m}\end{array}$ & $\boldsymbol{N}$ & Optimal \\
\hline 1 & Chr20a & 20 & 2192 & 9 & Tai60a & 60 & 7205962 \\
\hline 2 & Chr20c & 20 & 14142 & 10 & Tai60b & 60 & 608215054 \\
\hline 3 & Chr25a & 25 & 3796 & 11 & Tai80b & 80 & 818415043 \\
\hline 4 & Nug28 & 28 & 5166 & 12 & $\begin{array}{c}\text { Will10 } \\
0\end{array}$ & 100 & 273038 \\
\hline 5 & Kra32 & 32 & 88900 & 13 & $\begin{array}{c}\text { Skol0 } \\
0 a\end{array}$ & 100 & 152002 \\
\hline 6 & Tai35b & 35 & 28331 & 14 & $\begin{array}{c}\text { Skol0 } \\
0 b\end{array}$ & 100 & 153890 \\
\hline 7 & Lipa40a & 40 & 30930 & 15 & $\begin{array}{c}\text { Thol5 } \\
0\end{array}$ & 150 & 8133398 \\
\hline 8 & Tho40 & 40 & 24051 & & & & \\
\hline
\end{tabular}

population

Step 2: Generate two crossover points $\mathrm{cp} 1$ and $\mathrm{cp} 2$ randomly. Then, exchange two substrings, which defined by two positions, between parents $\mathrm{P} 1(4,5,6,7$ in Figure 4) and P2 (2, 8, 3, 4 in Figure 5) to product $\mathrm{C} 1$ and $\mathrm{C} 2$.

Step 3: Exchange the cells which are already in the substring from the parent $\mathrm{P} 2$ and $\mathrm{P} 1$, for example the second gene of C1:2->4->7.

Table 1: Benchmark functions (1-15)

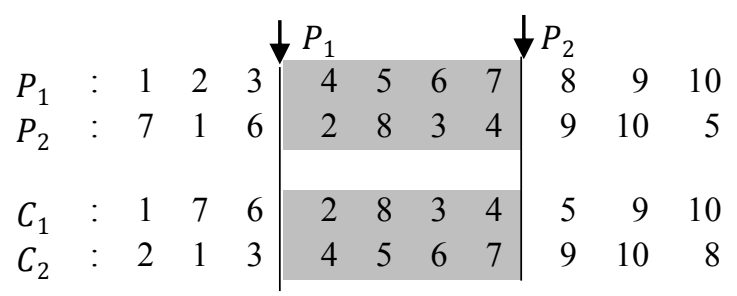

Figure 5: PMX process

For scout bee stage, we choose two different cells randomly in food source array and then exchange them. This operator is illustrated in figure 6.

\begin{tabular}{|l|cc|c|cc|c|cc|c|c|}
\hline Food & 2 & 5 & 10 & 7 & 8 & 3 & 1 & 4 & 6 & 9 \\
\hline New Food & 2 & 5 & 6 & 7 & 8 & 3 & 1 & 4 & 10 & 9 \\
\hline
\end{tabular}

\section{NUMERICAL RESULTS}

To verify the feasibility of the proposed algorithm to solve QAPs, some benchmarks problems were tested by using the software written in Matlab 8.1 programming languages and executed in the Intel Core 2 Duo 2.20 GHz. The results obtained for solving QAPs are compared with other algorithms.

The proposed $\mathrm{ABC}$ is tested using 15 benchmarks problems [24]. For comparison, Genetic Algorithm (GA) [28], and Simulated Annealing (SA) [7] are also executed on these 15 problems. Table 1 shows the details of test benchmark problems. The parameter settings of $\mathrm{ABC}$ algorithm are described as follows: number of bees NB is equal to 100 , number of iterations is equal to 500 and the limit parameter is equal to 1 . The algorithm is conducted 20 runs for each test problem.

In Table 2, the performance of $\mathrm{ABC}$ algorithm is compared with GA and SA using statistical measures (Mean, Maximum value, Minimum value, and Standard Deviation, Error). The Error of each algorithm can be calculated as:

$$
E_{(\text {Algorithm })}=100 \% *\left(1-\frac{\text { optimal }}{M_{(\text {Algorithm })}}\right)
$$

\section{CREATE QAP FOR AZADI HOSPITAL}

In this section, a real life quadratic assignment problem is presented. Azadi hospital in Kirkuk city was established in 1985 and it's currently provides a wide range of medical services, in a highest level under the supervision of the best specialist doctors who have high experience in different medical fields. In this paper, we create a QAP to Azadi hospital.

\section{A. Desicription of the hospital layout}

The hospital building has 6 floors in addition to the ground floor and its contains about 398 beds supported with new medical equipment's the hospital contain emergency room provided with emergent medical equipment and also contain operating room for major and minor operations with different types of laboratories for give the best medical care to all people.

The failure to regulate the clinics sites for some of them causes a lot of movements for patients, which in turn leads to obtain a serious delays. That is why the problem of determining the clinics location within the hospital is very important. The goal is to decide on the 
various clinics site to reduce the effort spent by patients while they moving from clinic to another, therefore the goal is to locate clinics within the hospital building to minimize the total distance to move patients through problem formulation as quadratic assignment problem.

The study applied on consultancy services (facilities) only located on the ground floor for 30 services. That is because the greater part of the patients coming to this floor of hospital every day. Table 2 shows consultative clinics (facilities) located in ground floor.

Table 2. Consultative Clinics

\begin{tabular}{|c|c|c|c|}
\hline$I$ & Service & $i$ & Service \\
\hline 1 & Entry & 16 & Neurological \\
2 & Pharmacy & 17 & lung functions \\
3 & Laboratory & 18 & Ultrasound \\
4 & Rays & 19 & Urologist \\
5 & Computerized Axial & 20 & pediatric \\
6 & Tomography & & Vagnetic Resonance \\
& 21 & \\
7 & Imaging & & General surgery \\
8 & Physical therapy & 23 & Trauma room \\
9 & Dentistry & 24 & Gynecology \\
10 & Ear Nose and Throat & 25 & The internists1 \\
11 & Ophthalmologic & 26 & The internists2 \\
12 & Endoscopy & 27 & Minor operations \\
13 & Allergy and Asthma & 28 & Interactions skin \\
14 & Echo & 29 & Dermatologic \\
15 & Straining the heart & 30 & Hematological \\
\hline
\end{tabular}

\section{B. Description of the data}

Estimates of the patient flows between clinics were available on a yearly basis. Entries in the flow matrix were obtained by averaging the flow between each pair of clinics. The distances between locations were actually measured by tracing the paths taken by patients while moving from one location to another. It was noticed that a patient, after being through a sequence of visits to more than one clinic, must go to the pharmacy to mark off his card. The distance matrix can be taken to be symmetric. The flow and distance data a can be compactly presented as in table 4 and table 5 respectively.

\section{Apply of QAP for Azadi Hospital}

At first, it should be noted that the study must be applied before starting the establishment of the hospital, because some of clinics need to special positions, i.e. Rays room needs his own bullets walls and some safety stuffs.

Therefore, some clinics must have been installed in their current positions because of the difficulty transferred to another places, such as pharmacy, laboratory and rays and so on. Moreover, the hospital has a consulting rooms and consulting departments, and the positions of department differ from those of rooms (i.e. ENT department has four rooms: audiogram room, two rooms for nose and throat doctors and ear doctor's room and Gynecology consulate has one room only).

Therefore, the problem will be divided consulting services (facilities) into three categories, the first category includes fixed consulting services, which must be installed for the lack of other similar places as entry, laboratory pharmacy, rays, computerized axial tomography, magnetic resonance imaging, electrocardiography and physical therapy. The second category represents consulting departments as dental department, ENT Department and Ophthalmology Department, while the third category represents consulting rooms like Echo, Straining the heart, Neurological, Lung functions, Ultrasound, Urologist, Pediatric, Vaccine, General surgery, Trauma room, Gynecology, The internists1, The internists2, Minor operations, Interactions skin, Dermatologic and Hematological.

Figure 7 shows the outline of the locations within the hospital, the red locations represents the first category and the blue locations represents the second category while the white category represents the third category accounting for sites in red Class I and Class II blue color while the white color represents the third category.

To know the distance traveled by the patients during their movement within the hospital, we use current permutations and calculate the cost function of QAP. The value is $(8,974,071)$ meter per month. To minimize this distance, we used proposed $\mathrm{ABC}$ algorithm to resolve the quadratic assignment problem for Azadi hospital, taking into consideration the clinics (facilities) that installed in the same location (First Category). While the second and third category represent the first and second problems which have been solved sequentially, The clinics of third category have been installed in their location when finding the best solution to the first problem and then we install the best solution for the first problem when solve the second problem, then install the solution of the second problem when solve the first problem. The process will continue until reach to a state of stability.

After steady state has been obtained, the cost of the best solution obtained is $(8,312,830)$ meter per month, as compared to the cost of the original layout which is $(8,974,071)$ meter per month, i.e. a resulting decrease of approximately $7.37 \%$. Figure 8 shows the scheme for the distribution of new sites for clinics (facilities) after solving the problem by using the proposed algorithm. 


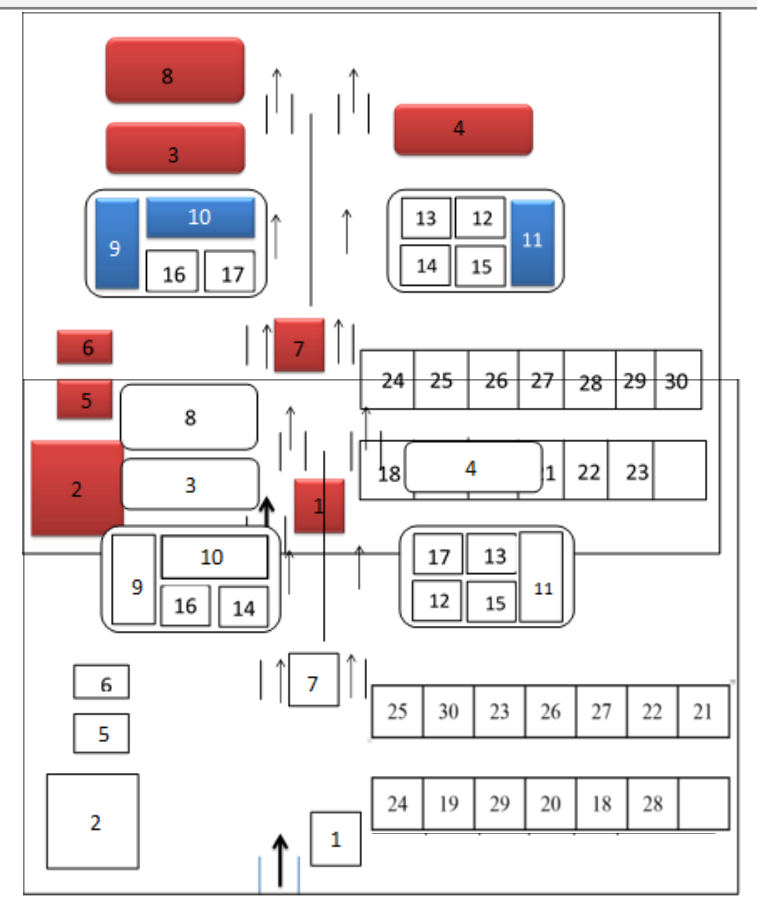

Figure 7: The outline of the locations within the hospital

Figure 8: The scheme for the distribution of new sites of clinics

\section{CONCLUSIONS}

This paper proposes a proposed Artificial Bee Colony (ABC) Algorithm for Quadratic Assignment Problem (QAP). The performance of $\mathrm{ABC}$ is evaluated using a set of well-known multi-dimensional benchmark functions. The results show the efficiency and capabilities of the proposed algorithm in finding the optimum. Amazingly, its performance is better than other algorithms such as $\mathrm{GA}$ and SA. The ABC described in this paper has led to an improvement of $7.37 \%$ in the total cost (expressed in terms of passenger meters travelled per month). Furthermore, the derived solution proved compatible with some medical considerations that management was keen to satisfy in the new layout.

\section{REFERENCES}

[1] Armour, G.C. and Buffa E.. Heuristic Algorithm and Simulation Approach to Relative Location of Facilities. Management Science, Volume 9, 1963.

[2] Bäck, T. (1996). "Evolutionary algorithms in theory and practice". New York: Oxford Univ. Press.

[3] Bazaraa M. S. and Sherali. H. D. On the Use of Exact and Heuristic Cutting Plane Methods for the Quadratic Assignment Problem. The Journal of the Operational Research Society, Volume 33, Issue 11, Novmber 1982.

[4] Behzadi, G. \& Sundarakani, B. 2014, 'Practical ABC intelligence

[5] solution for Quadratic Assignment Problems', 4th International conference on Industrial Engineering and Operations Management (IEOM), IEOM, Indonesia, pp. 959-966.
[6] Dell'Amico.M, Carlos Díaz.J, Iori.M, and Montanari.R,. (2009)." The single-finger keyboard layout problem".Computers and Operations Research, V( 36), Issue 11, Pages 3002-3012.

[7] Dickey, J.W. and Hopkins, J.W., .(1972)"Campus building arrangement using TOPAZ". Transportation Research 6, 59-68.

[8] Dowsland, K.A., .( 1995), "Simulated Annealing. In Modern Heuristic Techniques for Combinatorial Problems" (ed. Reeves, C.R.), McGraw-Hill

[9] Drezner, Z., .(2002)."Heuristic Algorithms for the Solution of the Quadratic Assignment Problem". Journal of Applied Mathematics and Decision Sciences 6, 163-173.

[10] Elshafei, A.N. (1977)."Hospital Layout as a Quadratic Assignment Problem". Operations Research Quarterly 28, 167179.

[11] Francis, R.L., White, J.A., .(1974)."Facility Layout and Location: An Analytical Approach". Prentice-Hall, Englewood Cliffs, NJ.

[12] Gilmore . P.C. Optimal and Suboptimal Algorithms for the Quadratic Assignment Problem. SIAM Journal on Applied Mathematics, Volume 10, 1962.

[13] Hahn, P. and Krarup, J., .(2001)."A hospital facility layout problem finally solved" ,Journal of Intelligent Manufacturing, Vol 12, 487-496.

[14] Hubert, L., .(1987)."Assignment methods in combinatorial data analysis". Statistics: Textbooks and Monographs Series, vol. 73. Marcel Dekker.

[15] Karaboga, D., Basturk, B. (2008). "On the performance of artificial bee colony (ABC) algorithm", Applied Soft Computing, Vol. 8, pp. 687-697.

[16] Karaboga, D.,Akay, B.,(2010)."Amodified Artifical Bee colony algorithm for real-Parameter optimization" Information Sciences and Elservier,doi:10.1016/j.ins.2010.07.015.

[17] Karboga, D. and Basturg B., (2007), "A powerful and efficient algorithm for numerical function optimization: artificial bee colony (ABC) algorithm", J. Glob. Optim. Vol. 39, pp.459-471.

[18] Kaur, A., Goyal, S., (2011), "A survey on the Applications of bee colony optimization Techniques", International Journal on Computer Science and Engineering (IJCSE), ISSN: 0975-3397 Vol. 3 No. 8.

[19] Koopmans, T.C., Beckmann, M.J., 1957."Assignment problems and the location of economic activities". Econometrica 25, 5376.

[20] Li Y., Pardalos P. M., and Resende M. G.C.. A Greedy Randomized Adaptive Search Procedure for the Quadratic Assignment Problem. DIMACS Series in Discrete Mathematics and Theoretical Computer Science.

[21] Loiola, E.M., de Abreu, N.M.M., Boaventuro-Netto, P.O., Hahn, P., and Querido, T., .(2007)." A survey for the quadratic assignment problem". European Journal of Operational Research 176, 657-690.

[22] Misevicius, A., .(2005)."A tabu search algorithm for the quadratic assignment problem". Computational Optimization and Applications ,30 (1), 95-111.

[23] Nehi, H. M. and Gelareh S.. A Survey of Meta-Heuristic Solution Methods for the Quadratic Assignment Problem. Applied Mathematical Sciences, Volume 1, Issue 46, 2007.

[24] Pollatschek, M.A., N. Gershoni, and Y.T. Radday (1976). "Optimization of the Typewriter Keyboard by Computer Simulation". Angewandte Informatik 10, 438-439.

[25] QAPLIB - A Quadratic Assignment Problem Library.(2012). Retrieved May 2014, from: http://www.seas.upenn.edu/qaplib

[26] Steinberg, L., 1961."The backboard wiring problem:A placement algorithm". SIAM Review 3, 37-50.

[27] Taha,H.A.,. (2007). "Operations Research An Introduction", Pearson Precintle hall, 8th Edition ,New Jersey, USA .

[28] Talbi E.-G., Roux O., Fonlupt C., and Robillard. D. Parallel Ant Colonies for the quadratic assignment problem. Future Generation Computer Systems, Volume 17 ,France, 2001. 
[29] Tate, D.E., Smith, A.E., .(1995)."A genetic approach to the quadratic assignment problem". Computers and Operations Research 22, 73-83.
[30] Wilhelm, M.R., Ward, T.L., (1987)."Solving quadratic assignment problems by simulated annealing". IEEE Transactions 19, 107-119. 